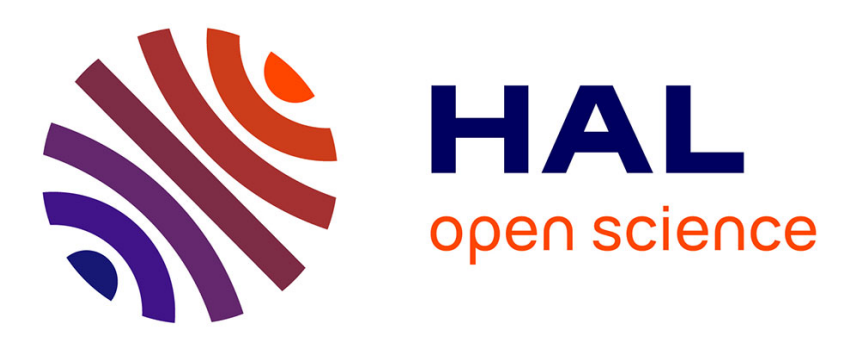

\title{
Receive Spatial Modulation for LOS mmWave Communications Based on TX Beamforming
}

Nemanja Stefan Perovic, Peng Liu, Marco Di Renzo, Andreas Springer

\section{To cite this version:}

Nemanja Stefan Perovic, Peng Liu, Marco Di Renzo, Andreas Springer. Receive Spatial Modulation for LOS mmWave Communications Based on TX Beamforming. IEEE Communications Letters, 2017, 21 (4), pp.921 - 924. 10.1109/LCOMM.2016.2642923 . hal-01880023

\section{HAL Id: hal-01880023 https://hal.science/hal-01880023}

Submitted on 7 Jul 2020

HAL is a multi-disciplinary open access archive for the deposit and dissemination of scientific research documents, whether they are published or not. The documents may come from teaching and research institutions in France or abroad, or from public or private research centers.
L'archive ouverte pluridisciplinaire HAL, est destinée au dépôt et à la diffusion de documents scientifiques de niveau recherche, publiés ou non, émanant des établissements d'enseignement et de recherche français ou étrangers, des laboratoires publics ou privés. 


\title{
Receive Spatial Modulation for LOS mmWave Communications Based on TX Beamforming
}

\author{
Nemanja Stefan Perović, Student Member, IEEE, Peng Liu, Member, IEEE, Marco Di Renzo, Senior Member, \\ IEEE and Andreas Springer, Member, IEEE
}

\begin{abstract}
Receive spatial modulation (RSM) is a recently introduced multi-antenna transmission scheme that is capable of achieving high spectral efficiency at low hardware and processing complexity. We study RSM for application in indoor line-of-sight (LOS) millimeter-wave (mmWave) communication. Significantly different from low-GHz indoor channels, which are typically modeled by a Rician distribution, indoor mmWave channels are sparsely-scattered and dominated by LOS propagation. Hence, we consider the specific characteristics of the LOS mmWave channel and identify the channel conditions that minimize the symbol error probability (SEP) of RSM. We prove that the SEP of RSM in a LOS channel is minimized by imposing orthogonality conditions to the channel matrix. In addition, we propose a simple hardware architecture for the transmitter (TX) which uses only RF phase shifters for precoding. Simulation results show that RSM can successfully operate in indoor mmWave channels.
\end{abstract}

Index Terms-Beamforming, line-of-sight (LOS) communication, receive spatial modulation (RSM).

\section{INTRODUCTION}

Receive spatial modulation (RSM) is a multi-antenna transmission scheme that belongs to the spatial modulation (SM) family [1]. RSM exploits the multiplicity of antennas available at the receiver (RX) as a spatial constellation diagram for improving the spectral efficiency at low complexity. In RSM, two data streams are transmitted in parallel: one in the in-phase/quadrature (IQ) domain by using conventional signal constellations, and the other in the spatial domain where the information is transmitted via the activated RX antenna indices, with the aid of transmitter (TX) precoding.

To date, research activities on SM/RSM have been mainly focused on their application at low-GHz frequencies [1]. RSM, in particular, was first introduced in [2] and then extended in [3], [4] into generalized RSM which supports more than one IQ data streams and shows similar or better error performance than spatial multiplexing (SMX).

The work of Peng Liu and Andreas Springer is partially supported by the Austrian COMET-K2 programme of the Linz Center of Mechatronics (LCM), and was funded by the Austrian federal government and the federal state of Upper Austria. The work of Marco Di Renzo is partially supported by the Agence Nationale de la Recherche Scientifique (ANR) through the research project SpatialModulation (Société de l'Information et de la Communication - Action Plan 2015)

Nemanja Stefan Perović, Peng Liu and Andreas Springer are with the Institute for Communications Engineering and RF-Systems (NTHFS), Johannes Kepler University (JKU), Linz, Austria (e-mail: \{nemanja_stefan.perovic, peng.liu, andreas.springer $\} @ j k u . a t)$.

M. Di Renzo is with the Laboratoire des Signaux et Systèmes, CNRS, CentraleSupélec, Univ Paris Sud, Université Paris-Saclay, 3 rue Joliot Curie, Plateau du Moulon, 91192, Gif-sur-Yvette, France (e-mail: marco.direnzo@12s.centralesupelec.fr).
Against this background, this letter is focused on the application of RSM in indoor millimeter-wave (mmWave) communications. This is a major novelty of our work since, so far, $\mathrm{SM} / \mathrm{RSM}$ has been studied only at low-GHz (below $6 \mathrm{GHz}$ ) frequencies [2], [3]. Indeed, to the best of our knowledge, the only paper that investigates the application of SM at mmWave frequencies is [5]. Therein, however, RSM was not considered.

At mmWave frequencies, e.g., $60 \mathrm{GHz}$, in particular, non-line-of-sight (NLOS) links are subject to high reflection losses in indoor environments, e.g., each reflection at the walls or the ceiling typically introduces $10 \mathrm{~dB}$ loss [6]. This implies that the signal propagation is dominated by line-of-sight (LOS) links. At low-GHz frequencies, on the other hand, indoor environments are characterized by rich scattering [7].

Motivated by the mentioned considerations, we study the optimization of RSM that takes the peculiarities of LOS channels at mmWave frequencies into account. The major contributions of this letter are as follows:

- The operating principle of RSM and SM [1]-[4] is based on encoding the information to be transmitted on the randomness of the channels. Common belief is that RSM may be unsuitable for application to mmWave frequencies due to the absence of rich scattering. We disprove this belief and show that the deterministic nature of LOS links at mmWave frequencies provides the opportunity to optimize the RSM system design. Relying on zeroforcing (ZF) precoding, we identify the optimal design condition that minimizes the symbol error probability (SEP) and allows RSM to outperform SMX systems.

- Due to signal processing complexity and power consumption issues, the realization of mmWave systems based on full digital implementations is considered impractical. Mixed analog/digital or full analog solutions are considered more practical and cost-effective. We show that the proposed optimal RSM encoding scheme can be realized by relying only on analog phase shifters. The proposed TX hardware architecture provides a low complexity yet optimal solution for RSM at mmWave frequencies. Also, we present a low complexity hardware architecture of the $\mathrm{RX}$ that achieves near-optimal error performance.

\section{SyStem And Signal Model}

1) System model: The RSM system is depicted in Fig. 1. Here we only introduce the system model. The RX signal vector at the output of the RX antennas can be written as

$$
\mathbf{y}=\mathbf{H} \mathbf{P} \mathbf{x}_{m}^{i} / \sqrt{N_{a}}+\mathbf{n},
$$


where $\mathbf{H} \in \mathbb{C}^{N_{r} \times N_{t}}$ and $\mathbf{P} \in \mathbb{C}^{N_{t} \times N_{r}}$ are the channel and precoding matrices. The noise vector $\mathbf{n} \in \mathbb{C}^{N_{r} \times 1}$ is distributed according to a circularly-symmetric complex Gaussian distribution $\mathcal{C N}\left(\mathbf{0}, N_{0} \mathbf{I}\right)$, where $N_{0}$ is the noise variance.

2) Channel model: In the considered system, the interantenna separations of the TX and RX antennas are $d_{t}$ and $d_{r}$, respectively. The TX-RX distance is denoted by $D$ and it is measured from the midpoint of the TX uniform linear array (ULA) to the midpoint of the RX ULA. We first neglect the NLOS components and optimize the RSM design based only the LOS components. The impact of NLOS components will be studied in Section V-3. The channel coefficient of the pure LOS channel between the $j$-th TX and the $i$-th RX antenna, normalized by the path loss due to $D$, is given by [5]

$$
h_{i j}=d_{i j} / D \cdot \exp \left(-\mathrm{j} k d_{i j}\right), \quad k=2 \pi / \lambda
$$

where $d_{i j}$ is the path length, and $\lambda$ is the wavelength.

3) Modulation and detection: The TX symbol vector at the input of the TX precoder is $\mathbf{x}_{m}^{i}=\mathbf{e}_{i} s_{m}$, where $i \in\{1, \ldots, N\}$ identifies the spatial symbol $\mathbf{e}_{i}$ and $m \in\{1, \ldots, M\}$ specifies the modulation symbol $s_{m}$. The spatial symbol $\mathbf{e}_{i}$ is a column vector that contains $N_{a}$ 1's and $N_{r}-N_{a}$ 0's. The positions of the 1's in $\mathbf{e}_{i}$ specify the indices of the RX antennas activated in the $i$-th RX antenna combination, which form the set $\Omega_{i}$. Each RX antenna combination consists of $N_{a}\left(N_{a}<N_{r}\right)$ active RX antennas. As a result, $\log _{2}\left(\left(\begin{array}{c}N_{r} \\ N_{a}\end{array}\right) M\right)$ bits per channel use can be transmitted, where the rate can be a fractional number and this can be achieved by fractional bits modulation [8].

The maximum likelihood (ML) (joint) detector of RSM [2], [3] is given as

$$
(\widehat{m}, \widehat{i})=\underset{\substack{m \in\{1, \ldots, M\} \\ i \in\{1, \ldots, N\}}}{\operatorname{argmin}}\left\|\mathbf{y}-\mathbf{H P x}_{m}^{i} / \sqrt{N_{a}}\right\|^{2} .
$$

4) Precoding: For ease of implementation, we consider ZFbased precoding [2], [3], i.e.,

$$
\mathbf{P}=\beta \mathbf{H}^{\mathrm{H}}\left(\mathbf{H H}^{\mathrm{H}}\right)^{-1},
$$

where

$$
\beta=\sqrt{N_{r} / \operatorname{Tr}\left(\left(\mathbf{H H}^{\mathrm{H}}\right)^{-1}\right)}
$$

is a scaling coefficient that ensures that $\mathbf{P}$ does not change the average power of the TX signal (i.e., $\operatorname{Tr}\left(\mathbf{P} \mathbf{P}^{\mathrm{H}}\right)=N_{r}$ ) and we assume $N_{t} \geq N_{r}$. From (1) and (4), the signals received by the activated $\mathrm{RX}$ antennas are

$$
y_{k}=\beta s_{m} / \sqrt{N_{a}}+n_{k}, \quad k \in \Omega_{i} .
$$

The other RX antennas contain only noise $y_{k}=n_{k}\left(k \notin \Omega_{i}\right)$.

\section{Optimization OF THE SEP}

In this section, we identify the operating conditions that minimize the SEP of ZF-RSM in LOS channels. Moreover, we provide simplified expressions of the SEP if the optimal design is fulfilled.

Based on the union bound [9] for ML (joint) detection, the SEP of ZF-RSM $P_{\mathrm{S}}$ is given as

$$
P_{\mathrm{s}} \leq \frac{1}{N M} \sum_{m=1}^{M} \sum_{\substack{n=1 \\ n \neq m}}^{M} \sum_{i=1}^{N} \sum_{\substack{j=1 \\ j \neq i}}^{N} Q\left(\sqrt{\Phi / 2 N_{0} N_{a}}\right),
$$

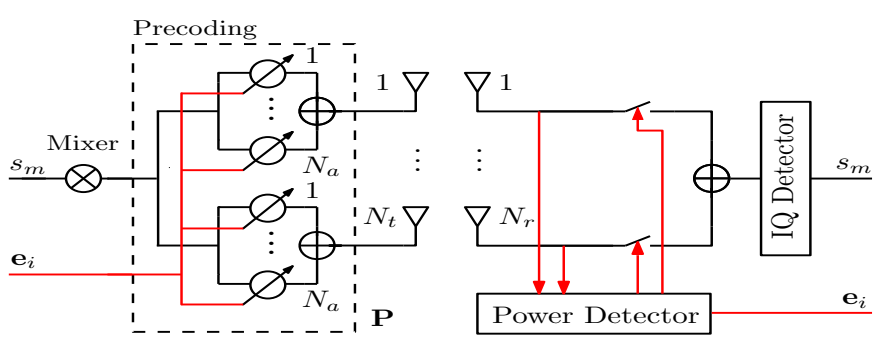

Fig. 1. Proposed hardware architecture for ZF-RSM.

where the Euclidean distance is $\Phi=\beta^{2}\left\|\mathbf{x}_{n}^{j}-\mathbf{x}_{m}^{i}\right\|^{2}$ and $N=\left(\begin{array}{l}N_{r} \\ N_{a}\end{array}\right)$. By inspection, we evince that $P_{\mathrm{s}}$ is minimized if $\Phi$ is maximized [9]. Since we do not consider any optimization of the signal modulation, the minimization of the SEP reduces to the design of the TX and RX such that the deterministic LOS channel matrix, $\mathbf{H}$, is

$$
\widehat{\mathbf{H}}=\arg \max _{\mathbf{H}} \beta^{2}\left\|\mathbf{x}_{n}^{j}-\mathbf{x}_{m}^{i}\right\|^{2} .
$$

From (8), the operating conditions that minimize the SEP of ZF-RSM in LOS channels are formulated in Theorem 1.

Theorem 1. Consider ZF-based RSM in LOS channels. The optimal strategy that minimizes the SEP is to make the rows of $\mathbf{H}$ orthogonal, i.e.,

$$
\left\langle\mathbf{h}_{i}, \mathbf{h}_{j}\right\rangle=0, i \neq j .
$$

Proof: We note that $\mathbf{H}$ affects only $\beta$ in (8), thus optimizing $\mathbf{H}$ results in maximizing $\beta$. Moreover, increasing $\beta$ increases the signal-to-noise ratio (SNR) at the RX and consequently reduces the SEP. Therefore, minimizing the SEP results in maximizing $\beta$ or, equivalently, minimizing $\operatorname{Tr}\left(\left(\mathbf{H} \mathbf{H}^{\mathrm{H}}\right)^{-1}\right)$ in (5). Thus, (8) can be re-written as

$$
\widehat{\mathbf{H}}=\arg \min _{\mathbf{H}} \operatorname{Tr}\left(\left(\mathbf{H} \mathbf{H}^{\mathrm{H}}\right)^{-1}\right) \text {. }
$$

By applying singular value decomposition (SVD) to $\mathbf{H}$ we obtain $\mathbf{H}=\mathbf{U} \boldsymbol{\Lambda} \mathbf{V}^{\mathrm{H}}$. Also, (10) can be written as

$$
\operatorname{Tr}\left(\left(\mathbf{H} \mathbf{H}^{\mathrm{H}}\right)^{-1}\right)=\operatorname{Tr}\left(\left(\mathbf{U} \boldsymbol{\Lambda} \mathbf{\Lambda}^{\mathrm{H}} \mathbf{U}^{\mathrm{H}}\right)^{-1}\right)=\sum_{i=1}^{N_{r}} \lambda_{i}^{-2} .
$$

The optimization problem is completely determined by the singular values $\lambda_{i}\left(i=1, \ldots, N_{r}\right)$ of $\mathbf{H}$. Accordingly, we have

$$
\operatorname{Tr}\left(\mathbf{H H}^{\mathrm{H}}\right)=\sum_{i=1}^{N_{r}} \sum_{j=1}^{N_{\mathrm{t}}}\left|h_{i j}\right|^{2}=\sum_{i=1}^{N_{r}} \lambda_{i}^{2}=N_{t} N_{r} .
$$

Note that in (12), we have used the fact that $d_{j i} / D \approx 1$, which is usually justified in indoor communications because of $d_{r}, d_{r} \ll D$. Equation (10) can be equivalently expressed as a function of $\left\{\lambda_{i}\right\}$ as

$$
\left(\hat{\lambda}_{1}, \ldots, \hat{\lambda}_{N_{r}}\right)=\operatorname{argmin} \sum_{i=1}^{N_{r}} \lambda_{i}^{-2} \text {, s.t. } \sum_{i=1}^{N_{r}} \lambda_{i}^{2}=N_{t} N_{r} .
$$

Using the method of Lagrange multipliers and the bordered Hessian matrix ${ }^{1}$, it follows that (13) is minimized if all the

${ }^{1}$ We consider $\left\{\lambda_{i}^{2}\right\}$ as the optimization variables, and the solution of the Lagrangian function is $(\forall i) \lambda_{i}^{2}=N_{t}$. To prove that this solution provides the minimum of (13), we need to show that the principle minors of the bordered Hessian matrix are negative which can be done by mathematical induction. 


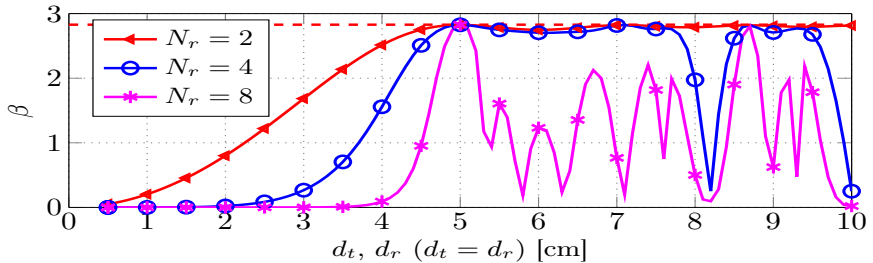

Fig. 2. Scaling coefficient $\beta$ versus inter-antenna separations $d_{t}, d_{r}\left(d_{t}=\right.$ $\left.d_{r}\right)$. The system setup is: $N_{t}=8, D=4 \mathrm{~m}$ and $\lambda=5 \mathrm{~mm}$. The dashed line denotes the maximum of $\beta$, which is $\beta_{\max }=\sqrt{N_{t}} \approx 2.83$.

singular values are $\lambda_{i}=\sqrt{N_{t}}$. If all the singular values are equal and denoted by $\lambda$, we get

$$
\mathbf{H H}^{\mathrm{H}}=\mathbf{U} \boldsymbol{\Lambda} \boldsymbol{\Lambda}^{\mathrm{H}} \mathbf{U}^{\mathrm{H}}=\lambda^{2} \mathbf{I} .
$$

Since all non-diagonal elements in (14) are 0, the rows of $\mathbf{H}$ are orthogonal. Thus, the operational design condition that minimizes the SEP is given by (9). From (9) the maximum value of $\beta$ is $\beta_{\max }=\sqrt{N_{t}}$.

If parallel ULAs in LOS channels are considered, the optimal condition in (9) reduces to [10]

$$
d_{t} d_{r} \approx D \lambda / N_{t},
$$

which presents the minimum value of $d_{t} d_{r}$ that satisfies (9). In Fig. 2, we show the change of $\beta$, computed according to (5), versus the inter-antenna separations $d_{t}=d_{r}$. From (15), the lowest $d_{t}=d_{r}$ for which $\beta$ achieves its maximum is $5 \mathrm{~cm}$, which is shown in Fig. 2. This confirms the optimality of (9) which maximizes $\beta$ and minimizes the SEP.

Finally, we provide a simplified expression of the SEP $P_{\mathrm{S}}$ of RSM when (9) is satisfied. Based on (7), $P_{\mathrm{s}}$ can be decomposed into the SEP of the IQ domain $P_{\text {iq }}$, the SEP of the spatial domain $P_{\mathrm{sp}}$ and the joint (of both domains) SEP $P_{\mathrm{j}}$, as $P_{\mathrm{s}} \leq P_{\mathrm{iq}}+P_{\mathrm{sp}}+P_{\mathrm{j}}$. Due to ZF-based precoding, the proposed scheme consists of $N_{a}$ orthogonal subchannels and each subchannel has an array gain $N_{t} / N_{a}$. So, after combining the signals from the activated RX antennas, the total array gain is $N_{t}$. If the symbol energy is $E_{s}$ and rectangular $M$-QAM is used, the SEP of the IQ domain $P_{\text {iq }}$ is

$$
P_{\mathrm{iq}}=1-R\left(M_{i}\right) R\left(M_{q}\right),
$$

where $R(M)=1-2(1-1 / M) Q\left(d / \sqrt{2 N_{0}}\right), M_{i}$ and $M_{q}$ are the signal levels in I and $\mathrm{Q}$, respectively, and $d=$ $\sqrt{12 N_{t} E_{s} /\left(M_{i}^{2}+M_{q}^{2}-2\right)}$. The joint SEP $P_{\mathrm{j}}$ is obtained from (7) by substituting in $\beta=\sqrt{N_{t}}$. Thus, we have

$$
\left.P_{\mathrm{j}}=\frac{1}{N M} \sum_{\substack{m, n, i, j \\(m \neq n, i \neq j)}} Q\left\|\mathbf{e}_{j} s_{n}-\mathbf{e}_{i} s_{m}\right\| \overline{\frac{N_{t}}{2 N_{0} N_{a}}}\right),
$$

where $m, n=1, \ldots, M$ and $i, j=1, \ldots, N$. The expression for the SEP of the spatial domain $P_{\mathrm{sp}}$ can be obtained from (17) by substituting in $m=n$.

\section{LOW-COMPlEXITY HARDWARE ARCHITECTURE}

In this section, we introduce a low complexity hardware implementation for the proposed transmission system which is depicted in Fig. 1. The proposed TX architecture is motivated by (9). The optimization criterion in (9) can be equivalently written as $\mathbf{H H}^{\mathrm{H}}=N_{t} \mathbf{I}$. Thus, we have

$$
\begin{aligned}
\mathbf{P} & =\beta \mathbf{H}^{\mathrm{H}}\left(\mathbf{H H}^{\mathrm{H}}\right)^{-1}=\mathbf{H}^{\mathrm{H}} / \sqrt{N_{t}} \\
& \approx\left[\exp \left(-\mathrm{j} \theta_{i j}\right)\right]^{\mathrm{T}} / \sqrt{N_{t}},
\end{aligned}
$$

where $\theta_{i j} \triangleq \angle h_{i j}$. In (18) we used the fact that $d_{j i} / D \approx 1$ (cf. Section III). All the elements of $\mathbf{P}$ have approximately unit magnitude and the proposed precoding method can be realized by using only phase shifters. The activation of a single RX antenna requires the activation of the proper column of $\mathbf{P}$. This is implemented by placing a phase shifter before each TX antenna whose phase shift values are determined by the elements of the corresponding column of $\mathbf{P}$. The activation of $N_{a} \mathrm{RX}$ antennas is realized by placing a group of $N_{a}$ parallel phase shifters before each TX antenna (i.e., $N_{t} N_{a}$ phase shifters in total). The factor $1 / \sqrt{N_{t}}$ in (18) implies that the signal power is equally distributed among all TX antennas and the factor $1 / \sqrt{N_{a}}$ in (1) implies that the signal power is equally distributed among $N_{a}$ parallel phase shifters.

In the proposed low-complexity RX architecture in Fig. 1, we detect the RX antenna combination and the IQ symbol separately in two steps [3], instead of doing it jointly, as the ML detector in (3) does. While the transmitted signal is located at the activate RX antennas, the signal power at these antennas is, in general, larger than the signal power at the inactive RX antennas ${ }^{2}$. Therefore, the active $N_{a} \mathrm{RX}$ antennas are detected based on maximum power detection as

$$
\left(\hat{i}_{1}, \ldots, \hat{i}_{N_{a}}\right)=\underset{i_{1}, \ldots, i_{N_{a}} \in\left\{1, \ldots, N_{r}\right\}}{\operatorname{argmax}} \sum_{i=i_{1}}^{i_{N_{a}}}\left|y_{i}\right|^{2} .
$$

The use of single-pole single-throw (SPST) switches at the RX ensures that only the signals from the RX antennas $\hat{i}_{1}, \ldots, \hat{i}_{N_{a}}$ are combined. After the RX signal combining we have $y_{r}=$ $\sum_{i} y_{i}=\sqrt{N_{a}} \beta s_{m}+\sum_{i} n_{i}$, where $i=\left\{\hat{i}_{1},, \ldots, \hat{i}_{N_{a}}\right\}$ and $\left\{y_{i}\right\}$ are given in (6). Thus, the IQ symbol is detected as

$$
\widehat{m}=\underset{m \in\{1, \ldots, M\}}{\operatorname{argmin}}\left|y_{r}-\sqrt{N_{a}} \beta s_{m}\right|^{2} .
$$

The separate detector in (19) and (20) has significantly lower computational complexity than the ML detector in (3), yet achieves near-optimal error performance (cf. Section V-3).

\section{Simulation Results}

In this section, we present the SEP of the proposed ZF-RSM scheme in a pure LOS channel. Beamforming (BF)-MIMO [5] and SMX-MIMO ${ }^{3}$ are used as benchmarks for performance comparison. Then we show the impact of NLOS components on the symbol error rate (SER) of RSM in the multipath indoor mmWave channel. Finally, we study the SER performance of RSM with the separate detector. In all cases we use the proposed TX architecture which requires only the knowledge of the LOS signal components for the TX precoding.

\footnotetext{
${ }^{2}$ Noise also influences the signal power at each RX antennas and thus can sometimes cause a deviation from the mentioned rule.

${ }^{3}$ For SMX based on ZF precoding and $N_{r}$ orthogonal subchannels, the SEP is, for rectangular $M$-QAM, $P_{\mathrm{S}}=1-\left[R\left(M_{i}\right) R\left(M_{q}\right)\right]^{N_{r}}$, where $d=$ $\sqrt{12 N_{t}\left(E_{s} / N_{r}\right) /\left(M_{i}^{2}+M_{q}^{2}-2\right)}$.
} 


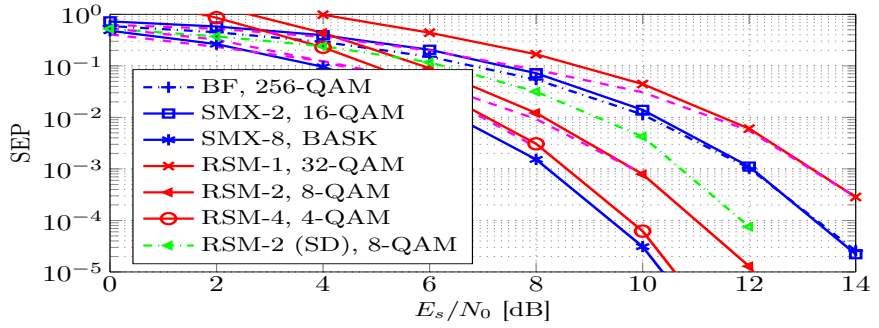

Fig. 3. SEP of RSM $(8 \times 8)$ in pure LOS channel.

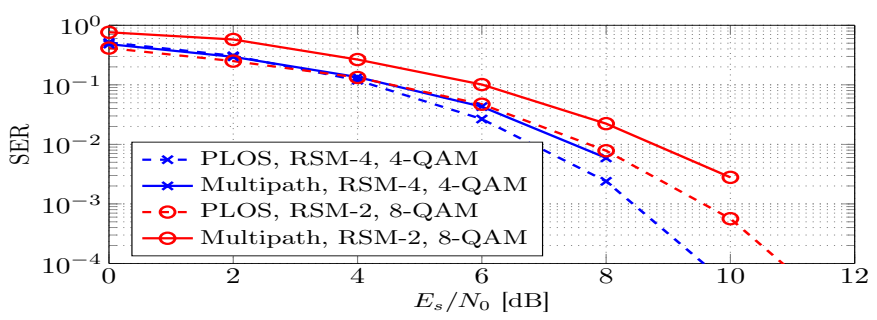

Fig. 4. SER of RSM $(8 \times 8)$ : multipath channel vs. pure LOS (PLOS) channel.

1) Error Performance of RSM with ML (joint) detection in pure LOS channels: We consider an $8 \times 8$ system for beamforming MIMO (BF-MIMO) and RSM. RSM with $N_{a}$ active RX antennas is denoted as RSM- $N_{a}$, where $N_{a} \leq N_{r} / 2$. The data rate of RSM- $N_{a}$ is maximized when $N_{a}=N_{r} / 2$. For fairness, we consider two configurations of SMX, i.e., $8 \times 2$ and $8 \times 8$, which are denoted by SMX-2 and SMX- 8 , respectively. The first configuration transmits 2 data streams, which is the same as for RSM, but requires fewer RX antennas than RSM. The second configuration, on the other hand, transmits 8 data streams, which is different from RSM, but employs the same number of RX antennas as RSM.

In Fig. 3, we show the SEPs of RSM, BF-MIMO and SMX in pure LOS channels at a data rate of 8 bpcu (small deviations occur due to non-integer data rates of RSM). In simulations, the inter-antenna separations are $d_{t}=d_{r}=5 \mathrm{~cm}$ for RSM and SMX, and $d_{t}=d_{r} \approx \lambda / 2=2.5 \mathrm{~mm}$ for BF-MIMO. All schemes have the same TX-RX ULA distance $D=4 \mathrm{~m}$. The simulated SER of RSM is depicted using dashed magenta curves. If $N_{a}>1$, RSM achieves better SEP than BF-MIMO and SMX-2. Only SMX-8 shows a small SEP advantage in comparison to RSM-4. However, the TX of SMX-8 has a higher hardware complexity than the TX of RSM. The TX of SMX requires $N_{t}$ modulators and up-converters, and the TX precoder of SMX needs $N_{t} N_{r}$ phase shifters.

2) Impact of NLOS components on the error performance of RSM with ML (joint) detection: We use the same multipath room channel model as [10, Fig. 4], which is based on raytracing and contains up to second order reflections. All NLOS components are modeled as in [6]. The differences in configuration are as follows. We considered a $8 \times 8$ system. Both ULAs are parallel to the $x$-axis and with an inter-array separation of $5.6 \mathrm{~cm}$. The midpoints of the ULAs are placed at $(2.5,0.1,2.5)$ and $(2.5,4.9,1)$, respectively, thus we have $D=5.02 \mathrm{~m}$. As we consider $1 \mathrm{GHz}$ wide-band single-carrier transmission, the NLOS paths introduce considerable intersymbol interference
(ISI) (tens of symbol durations). To handle this issue, we use a single-carrier frequency domain equalization (SC-FDE) reception algorithm, which was introduced in [11] to handle the ISI issue of RSM/SM systems. The simulation results are depicted in Fig. 4. Despite the additional computational complexity of FDE, worse error performance is obtained over the multipath channel as compared to the pure LOS channel. As it is necessary that the TX and RX have simple hardware architectures and low computational complexities in mmWave communications, we propose the use of the TX and RX from Fig. 1 together with directional antennas in order to suppress the NLOS components.

3) Performance of RSM with separate detection: The SER of the proposed RX is plotted in Fig. 3 (green curve). In comparison to the SER of RSM with the ML (joint) detector, the separate detector provides $1-2 \mathrm{~dB}$ worse SER. Similar performance trends can be shown for RSM-1 and RSM-4 (omitted due to space limitations). As a result, the SER performance penalty of the separate detector is tolerable.

\section{CONCLUSION}

In this letter, we have studied RSM for application to LOS mmWave communications. We derived the optimal operating conditions that minimize the SEP of RSM in LOS channels. Also, based on the identified optimization criterion, we proposed a simple TX and RX hardware architecture. The proposed TX uses only phase shifters for realizing TX precoding and the proposed RX offers near-optimal SER. Simulation results show that RSM is a good candidate for application to mmWave indoor communications.

\section{REFERENCES}

[1] M. Di Renzo et al., "Spatial modulation for generalized MIMO: Challenges, opportunities, and implementation," Proc. IEEE, vol. 102, no. 1 , pp. 56-103, Jan. 2014.

[2] L.-L. Yang, "Transmitter preprocessing aided spatial modulation for multiple-input multiple-output systems," in Vehicular Technology Conf. (VTC Spring), 2011 IEEE 73rd, May 2011, pp. 1-5.

[3] R. Zhang et al., "Generalised pre-coding aided spatial modulation," IEEE Trans. Wireless Commun., vol. 12, no. 11, pp. 5434-5443, Nov. 2013.

[4] A. Stavridis et al., "Performance analysis of multistream receive spatial modulation in the MIMO broadcast channel," IEEE Trans. Wireless Commun., vol. 15, no. 3, pp. 1808-1820, Mar. 2016.

[5] P. Liu et al., "Line-of-sight spatial modulation for indoor mmwave communication at $60 \mathrm{GHz}$," IEEE Trans. Wireless Commun., vol. 15 , no. 11, pp. 7373-7389, Nov. 2016.

[6] A. Maltsev et al., "Channel Models for $60 \mathrm{GHz}$ WLAN Systems," Tech. Rep. IEEE 802.11-09/0334r8, May 2010. [Online]. Available: https://mentor.ieee.org/802.11/dcn/09/11-09-033408-00ad-channel-models-for-60-ghz-wlan-systems.doc

[7] N. Serafimovski et al., "Practical implementation of spatial modulation," IEEE Trans. Veh. Technol., vol. 62, no. 9, pp. 4511-4523, Nov. 2013.

[8] - "Fractional bit encoded spatial modulation (FBE-SM)," IEEE Commun. Lett., vol. 14, no. 5, pp. 429-431, May 2010.

[9] M. Di Renzo and H. Haas, "Bit error probability of SM-MIMO over generalized fading channels," IEEE Trans. Veh. Technol., vol. 61, no. 3, pp. 1124-1144, Mar. 2012.

[10] E. Torkildson et al., "Indoor millimeter wave MIMO: Feasibility and performance," IEEE Trans. Wireless Commun., vol. 10, no. 12, pp. 41504160, Dec. 2011.

[11] S. Sugiura and L. Hanzo, "Single-RF spatial modulation requires singlecarrier transmission: Frequency-domain turbo equalization for dispersive channels," IEEE Trans. Veh. Technol., vol. 64, no. 10, pp. 4870-4875, Oct. 2015. 